\title{
Rate Theory on Water Exchange in Aqueous Uranyl Ion
}

\author{
Liem X. Dang \\ Physical Sciences Division \\ Pacific Northwest National Laboratory, \\ Richland, WA 93352 \\ and
}

Quynh N. Vo, Mikael Nilsson, and Hung D. Nguyen

Department of Chemical Engineering and Materials Science

University of California

Irvine, CA 92697 


\begin{abstract}
We report a classical rate theory approach to predict the exchange mechanism that occurs between water and aqueous uranyl ion. Using our water and ion-water polarizable force field and molecular dynamics techniques, we computed the potentials of mean force for the uranyl ionwater pair as a function of different pressures at ambient temperature. These potentials of mean force were used to calculate rate constants using transition rate theory; the transmission coefficients also were examined using the reactive flux method and Grote-Hynes approach. The computed activation volumes are positive; thus, the mechanism of this particular water-exchange is a dissociative process.
\end{abstract}




\section{Introduction}

Understanding the behavior of aqueous complexes such as uranyl ions in the condensed phase is fundamentally and technologically important because of its direct relevance to the nuclear fuel cycle. Knowledge of solvent-exchange and the kinetic properties of aqueous uranyl ions is essential for the development and improvement of solvent extraction processes used to recover these ions from spent nuclear fuels.[1-5]

Significant progress has been made in this particular research area. Wipff and coworkers generated force field parameters for uranyl ions-water from free energy calculations, and reported on molecular dynamics (MD) simulation studies of the complexation and hydration behavior of aqueous uranyl ions.[6] More recently, Kerisit and Liu modified Wipff's ion-water potential parameters and carried out studies on the structure, free energy, and kinetics of the uranyl ion using a variety of theoretical methods.[7] Maginn and co-workers developed classical force field parameters for aqueous actinyl cations using quantum mechanical calculations and validated their results against static properties as well as the dynamical properties of the wateractinyl ion system.[8]

The main goal of our work is to advance the understanding of the water-exchange mechanism around the actinyl ion. Knowledge of free energy profiles and rate theory evaluations are important for understanding a wide range of physical and chemical phenomena for these ionic systems. Information from those sources also provides a challenging test of the accuracy of the information derived from force field models. Our work is distinguished from earlier contributions by the methodology and to the extent to which we have exploited rate theory approaches. The most important values of this work are (1) polarization effects are explicitly included in the potential models, (2) pressure dependence and solvent response to the rate 
constant were are evaluated, and (3) activation volume can be calculated from the rate constants as the function of pressures and this quantity can be compared directly to the experimental measurements. The remainder of this paper is organized as follows. In Section II, we describe the potential models and simulation methods used. Results and discussion are presented in Section III, and our conclusions discussed are in Section IV.

\section{Potential Models, Simulations, and Methods}

For water-water interactions, we employed the Dang-Chang (DC) polarizable water model.[9] Because of uncertainties in the hydration number and hydration energy,[7, 8] we expended substantial effort in developing the polarizable force field parameters for $\mathrm{UO}_{2}{ }^{2+}$-water interactions. The functional form that describes the $\mathrm{UO}_{2}{ }^{2+}$-water interactions is the same as used in the DC model as shown below. The total interaction energy of the system is summarized as follows:

$$
U_{\text {tot }}=U_{\text {pair }}+U_{\text {pol }}
$$

$U_{\text {pair }}=\sum_{i} \sum_{j>i}\left(4 \varepsilon_{i j}\left[\left(\frac{\sigma_{i j}}{r_{i j}}\right)^{12}-\left(\frac{\sigma_{i j}}{r_{i j}}\right)^{6}\right]+\frac{q_{i} q_{j}}{r_{i j}}\right)$

and

$$
U_{p o l}=-\sum_{i=1}^{N} \mu_{i} \bullet E_{i}^{0}-\frac{1}{2} \sum_{i=1}^{N} \sum_{j=1, i \neq j}^{N} \mu_{i} \bullet T_{i j} \bullet \mu_{j}+\sum_{i}^{N} \frac{\left|\mu_{i}\right|^{2}}{2 \alpha_{i}}
$$

Here, $\mathrm{r}_{\mathrm{ij}}$ is the distance between site $\mathrm{i}$ and $\mathrm{j}, \mathrm{q}$ is the charge, and $\sigma$ and $\varepsilon$ are the Lennard-Jones parameters, $\boldsymbol{E}_{i}^{o}$ is the electric field at site i produced by the fixed charges in the system, $\boldsymbol{\mu}_{\mathrm{i}}$ is the induced dipole moment at atom site $\mathrm{i}$, and $\boldsymbol{T}_{\mathrm{ij}}$ is the dipole tensor. The first term in Eq. (3) represents the charge-dipole interaction, the second term describes the dipole-dipole interaction, and the last term is the energy associated with the generation of the dipole moment $\boldsymbol{\mu}_{\mathrm{i}}$. During 
molecular dynamics simulations, a standard iterative self-consistent field procedure is used to evaluate the induced dipoles.

We performed several MD simulations to optimize the Lennard-Jones potential and the polarizability parameters for the $\mathrm{UO}_{2}{ }^{2+}$ molecule. The final parameters that reproduce the experimental hydration number and hydration enthalpy are summarized in Table 1, Table 2, and Figure 1.

Equation 4 was used to calculate the ion-water mean force as an average over the different solvent configurations:[10]

$$
F(r)=\frac{1}{2}\left\langle\vec{r}_{u} \bullet\left(\vec{F}_{A}-\vec{F}_{B}\right)\right\rangle
$$

In this expression, $F_{A}$ and $F_{B}$ are the forces acting on the solutes. The term, $\vec{r}_{u}$, which is a unit vector along the $\mathrm{A}-\mathrm{B}$ direction, is defined as:

$$
\vec{r}_{u}=\vec{r}_{A B} / r_{A} \quad r_{B} \mid
$$

The potential of mean force (PMF), $W(r)$, is calculated as:

$$
W(r)=-\int_{r_{o}}^{r_{s}}\langle F(r)\rangle d r
$$

We evaluated PMFs along the center-of-mass separation between the $\mathrm{UO}_{2}{ }^{2+}-\mathrm{H}_{2} \mathrm{O}$ interactions with the separation between them incremented by $0.1 \AA$. At each center-of-mass separation, the average $F(r)$ was determined from a 2-ns simulation time, preceded by a 500 -ps equilibration period. The uncertainties of the PMFs were $\pm 0.05 \mathrm{kcal} / \mathrm{mol}$ as estimated by determining the force averaged (the corresponding PMFs) over four equally spaced time frames during the production. The systems investigated consisted of one $\mathrm{UO}_{2}{ }^{2+}$ in 1066 water molecules. All simulations were performed in an NVT ensemble at $300 \mathrm{~K}$, with periodic boundary conditions applied in all three directions and a time step of 2 fs. To compute the $\Delta \mathrm{V}^{\ddagger}$, we carried out three studies at pressures 
of 0,2 , and 4 kbar, which correspond to cubic box lengths of $31.8,31.2$, and $30.7 \AA$, respectively. We used a modified version of the Amber 9 software package to perform all MD simulations;[11] the Ewald summation technique to handle long-range electrostatic interactions;[12] and the SHAKE algorithm to fix the internal water and $\mathrm{UO}_{2}{ }^{2+}$ geometries.[13]

\section{Results and Discussions}

We start with the PMFs for the $\mathrm{UO}_{2}{ }^{2+}-\mathrm{H}_{2} \mathrm{O}$ pair at pressures of 0,2 , and $4 \mathrm{kbar}$, and then continue with rate theory results using the transition state theory (TST),[14] reactive flux (RF), [15] Grote-Hynes (GH), [16] and Impey, Madden, and McDonald (IMM) methods.[17] We end the section with the computed activation volume, $\Delta \mathrm{V}^{\ddagger}$. Figure 2 shows the computed PMF values obtained at $300 \mathrm{~K}$ for the three pressures normalized to the contact $\mathrm{UO}_{2}{ }^{2+}-\mathrm{H}_{2} \mathrm{O}$ pair free energy minimum at 0 bar. As expected, the shapes of the computed PMFs are very similar, and the changes are small but noticeable. We observe two effects: (1) an increase in pressure stabilizes the contact $\mathrm{UO}_{2}{ }^{2+}-\mathrm{H}_{2} \mathrm{O}$ pair and (2) the free energy barrier for escaping the first hydration shell increases from $6.82 \pm 0.05 \mathrm{kcal} / \mathrm{mol}$ at 0 bar to $7.20 \pm 0.05 \mathrm{kcal} / \mathrm{mol}$ at $4 \mathrm{kbar}$. These changes are accompanied by a small change in the transition state distance from $3.25 \AA$ to $3.24 \AA$. For a given PMF, the rate constant for the exchange process can be computed using TST as follows:[14]

$$
\boldsymbol{k}^{T S T}=\sqrt{\frac{\boldsymbol{k}_{\boldsymbol{b}} \boldsymbol{T}}{2}} \frac{(\boldsymbol{r})^{2} e^{W(r)}}{{ }_{0}^{r} r^{2} e^{W(r)} d r}
$$

where $r$ is defined as the position of the barrier top, $\quad$ is the ion-water reduced mass, $k_{b}$ is the Boltzmann constant, and $T$ is the temperature. Using the computed PMFs and transition state distances, we computed the rate constant, $k^{\mathrm{TST}}$, for the exchange process using Equation 7; the results for $k^{\mathrm{TST}}$ are $1.48 \times 10^{-4}, 1.09 \times 10^{-4}$, and $0.85 \times 10^{-4} \mathrm{ps}^{-1}$ at 0,2 , and $4 \mathrm{kbar}$, 
respectively. These results are summarized in Table 3 . We found $k^{\mathrm{TST}}$ increases with increasing pressure, and this trend would be expected by examining at the PMFs and the barrier heights. Equation 8 gives the pressure dependence of the rate constant at constant temperature.[18]

$$
\Delta \mathrm{V}^{*}=R T \frac{\ln (k)}{P}{ }_{T}
$$

where $\Delta \mathrm{V}^{*}$ is the activation volume, $\mathrm{T}$ is the temperature, $\mathrm{R}$ is the gas constant, $k$ is the rate constant, and $P$ is the pressure. An approximate solution to Equation 8 can be obtained using Equation 9:

$$
\ln \frac{k_{p}}{k_{0}} \div=-\Delta \mathrm{V}^{*} \frac{\mathrm{P}}{\mathrm{RT}}
$$

We use Equation 9 to calculate the activation volumes, where $k_{p}$ and $k_{0}$ are the rate constants at pressures $P$ and 0 , respectively. When the computed rate constants were used, a positive activation volume $\left(3.5 \mathrm{~cm}^{3} / \mathbf{m o l}\right)$ was obtained. These results establish that an exchange process with increasing pressure and increasing free energy at the barrier will lead to a positive activation volume (i.e., a dissociative mechanism).[18]

TST is known to significantly overestimate the values of rate constants. Because of the assumption that once the reactive species reach the transition state, they directly end up on the products side of the reaction without re-crossing to the reactants side. The RF method and GH theory are well known and popular corrections to TST that have been reported in the literature. $[15,16]$

The RF method and GH theory approaches provide a transmission coefficient, $\kappa$, to correct the TST. In the RF method, the transmission coefficient, $\kappa_{\square \square}$, is extracted from the plateau value of the time-dependent transmission coefficient as calculated using Equation 10: 


$$
k(t)=\frac{\left\langle\dot{r}(0)\left[r(t) \quad r^{*}\right]\right\rangle_{c}}{\langle\dot{r}(0)[\dot{r}(0)]\rangle_{c}},
$$

where $(x)$ is the Heaviside function, which is 1 if $x$ is larger than 0 and 0 otherwise, and $\dot{r}(0)$ is the initial ion-water velocity along the reaction coordinate. The subscript $c$ means that the initial configurations have been generated in the constrained reaction coordinate ensemble. We constrained the reaction coordinate at its transition state value in the RF and GH approaches by removing the center-of-mass motion of each $\mathrm{UO}_{2}{ }^{2+}$ and water molecules that were pre-selected to form a solute pair to perform the kinetics study in solution. To compute the time dependence transmission coefficient, we generated a series of starting configurations by running a simulation in which the distance between the $\mathrm{UO}_{2}{ }^{2+}$ and a selected water molecule was constrained to be the transition state distance. We carried out a 10-ns simulation was carried out, and collected a configuration was collected every 4 ps to obtain 2500 configurations. Then, we ran each configuration both backward and forward for $2 \mathrm{ps}$, and the value of ${ }_{R F}$ was determined by averaging $\kappa(t)$ over the last $0.5 \mathrm{ps}$ of the forward and backward rate constants. In Figure 3, we present the computed time-dependent $\kappa(t)$ at three different pressures. It is clear that the results converted well, and we observed that the rate constants decrease as pressure increases. The transmission coefficients ${ }_{R F}$, estimated as described above, are $2.1 \times 10^{-1}, 2.2 \times 10^{-1}$, and $2.3 \times$ $10^{-1}$. The activation volume using the correct rate constants is $3.3 \mathrm{~cm}^{3} / \mathrm{mol}$, which has the same sign as the value extracted using $k^{\mathrm{TST}}$. Thus, we conclude that the pressure dependence of both the barrier height and the transmission coefficient contribute minimally to the activation volume.

As mentioned above, we were interested in using GH theory to compute the exchange kinetic properties, such as the values for ${ }_{G H}$, of the $\mathrm{UO}_{2}{ }^{2+}-\mathrm{H}_{2} \mathrm{O}$ pair, and then comparing the values with corresponding results obtained using the RF method and with experimental values. 
To accomplish this task, we computed the friction kernel using the trajectories at the barrier region in Equations 11 and 12:

$$
\begin{gathered}
(t)=\frac{1}{k_{b} T}\langle R(t, r) \times R(0, r)\rangle \\
R(t, r)=F(t, r) \quad\langle F(t, r)\rangle
\end{gathered}
$$

where $r$ is the position of the barrier maximum, is the reduced mass, $k_{b}$ is the Boltzmann constant, and $T$ is the temperature. The GH theory transmission coefficient that accounts for re-crossings at the barrier, ${ }_{G H}$, can be expressed using Equation 13.[15]

$$
{ }_{G H}={ }_{G H}+{ }_{0} d t \frac{(t)}{b} e^{b G H^{t}} \stackrel{1}{\leftarrow}
$$

The GH theory transmission coefficient involves the frequency component of the timedependent friction coefficient, $(t)$, at the Laplace frequency, ${ }_{b} G H$, relevant in the barrier region. The ${ }_{b}$ term is the barrier frequency obtained by fitting the PMF in the barrier region to an inverted parabola. Figure 4 shows the un-normalized, time-dependent friction kernels of the $\mathrm{UO}_{2}{ }^{2+}-\mathrm{H}_{2} \mathrm{O}$ pair as the function of pressures. In all cases, there are two distinct decay time scales; the first one rapidly decay for about $0.1 \mathrm{ps}$, and the second time scale lasts for a few picoseconds with a longer decay time. We can conclude from these data that the oscillating $(t)$ reflects the barrier heights of the computed PMFs. We observed that the value of $\quad{ }_{b}$ decreases from $27 \mathrm{ps}^{-1}$ to $25 \mathrm{ps}^{-1}$ as the system pressure increases from 0 bar to $4 \mathrm{kbar}$. Transmission coefficients computed using GH theory are provided in Table 3. The computed values for ${ }_{G H}$ using these results are $2.4 \times 10^{-1}, 1.5 \times 10^{-1}$, and $1.3 \times 10^{-1}$ for three pressures, respectively. The activation volume using the computed ${ }_{G H}$ value is $7.3 \mathrm{~cm}^{3} / \mathrm{mol}$, which has the same sign as the value 
extracted using $k^{\mathrm{TST}}$. However, its value is significantly larger, indicating that solvent effects are of importance.

In addition to the RF and GH methods, we also used the IMM method in our study.[17] The IMM method allows the residence time of a solvent molecule in the first solvation shell of another molecule (i.e., $\mathrm{UO}_{2}{ }^{2+}$ ) to be calculated, and the inversion of the residence time is the rate constant. We used Equation 14 to determine the normalized time-correlation function of the population of solvent molecules in the first solvation shell of the $\mathrm{UO}_{2}{ }^{2+}$.

$$
P(t)=<n_{\alpha}\left(t_{i}, t_{f}, t^{*}\right)>/<n_{\alpha}\left(t_{i}, t_{i}, t^{*}\right)>_{\alpha, t_{i}}
$$

The term $n_{\alpha}$ is number of solvent molecules. It is assigned a value of 1 if a solvent molecule is found in the first solvation shell at both the initial time, $t_{i}$, and the final time, $t_{f}$, and if it does not leave the shell for a continuous period of time longer than $t^{*}=2 \mathrm{ps}$. To ignore transient escapes from the first solvation shell, $t^{*}$ is used. The function $\langle\cdots\rangle_{\alpha, t_{i}}$ in Equation 14 indicates averaging over solvent molecules, $\alpha$, and initial times, $t_{i}$. The term $P(t)$ is approximated as the exponential decay function $\exp \left(-t / \tau_{\mathrm{p}}\right)$ so the residence time, $\tau_{\mathrm{p}}$, can be determined using Equation 15:

$$
\tau_{\mathrm{p}}=\int_{0}^{\infty} P(t) d t=\int_{0}^{\infty} \exp \left(-t / \tau_{\mathrm{p}}\right) d t
$$

In this study, we used $t^{*}=2.0$ ps (i.e., the value originally proposed by Impey et al.).[17] In Figure 5, we show the values of $P(t)$ as a function of the pressure obtained using the IMM method. The decay rate of $P(t)$ is much slower for the $\mathrm{H}_{2} \mathrm{O}$ system at higher pressures than at lower pressures. This result would be expected because the fluid is denser at higher pressures. This finding essentially means that the value of $\tau_{\mathrm{p}}$ for $\mathrm{H}_{2} \mathrm{O}$ in the first solvation shell of the $\mathrm{UO}_{2}{ }^{2+}$ (i.e., $\tau_{\mathrm{p}}$ ) is smaller at lower pressures. This is justified quantitatively by integrating $P(t)$, which gives us $\tau_{\mathrm{p}}$ values of 15,27 , and $29 \mathrm{~ns}$ for pressures of 0,2 , and $4 \mathrm{kbar}$, respectively. The 
corresponding rate constants are $6.8 \times 10^{-5}, 3.7 \times 10^{-5}$ and $3.4 \times 10^{-5} / \mathrm{ps}$. A comparison of the computed resident times for $\mathrm{UO}_{2}{ }^{2+}$ in water using the $\mathrm{RF}, \mathrm{GH}$, and IMM methods are provided in Table 3. The observed trends are very consistent; however, the numerical values obtained using the IMM method are consistently higher.

We end this section by discussing the performance of the different rate theory methods. Throughout this study, we have recognized the value of the rate theory approach. With the correction provided in Table 3, agreement with the experimental results reported by Grenthe and co-workers[19] using ${ }^{17} \mathrm{O}$ NMR measurements $\left(k=1.4 \times 10^{6} / \mathrm{s}\right)$ is significantly improved. One of the shortcomings of our MD simulations (i.e., values a factor of 10 less than found in experimental data) is probably due to our choice of the reaction coordinate. Maginn and coworkers [8] discussed the shortcomings of this system in their recently published paper. We realize that the value of the rate constant is quite small (i.e. $k=0.2$ ) because of the significant amount of recrossings. We are planning to extend the rate theory approach to investigate rare events of water exchange by exploring many different metastable states of the ion-solvent coordination number.[20] One of the alternate reaction coordinates could be defined as the number of solvent molecules in the first solvation shell of a uranyl ion. This approach can improve the recrossing issue and, therefore, may improve the rate theory results. As mentioned above, our molecular models for water molecules and uranyl ion are nonflexible, which may contribution to the simulation shortcomings. 


\section{Conclusion}

Through MD simulations using polarizable force field models, we studied in detail the water-exchange mechanism around aqueous uranyl ions. We computed PMFs and estimated rate constants using three different rate theories: TST, GH theory, and the RF method. In addition, we employed the IMM method to compute the residence time directly and the corresponding exchange rate constants. We investigated the pressure dependence of rate constants to understand the water-exchange mechanism in the first solvation shell.

Our findings show that the free energy barrier heights increase as pressure increases, and as a result, the TST rate constants decrease as pressure increases. Transmission coefficients computed using GH theory and the RF method increase as pressure increases. Rate constants calculated using GH theory and the RF method decrease as pressure increases. The activation volume, which is a key indicator of the exchange mechanism, was found to be positive from TST, indicating a dissociative mechanism for this particular uranyl ion. Similar findings from the use of GH theory and the RF method yield positive activation volumes, thus indicating a dissociative mechanism. 


\section{Acknowledgments}

The U.S. Department of Energy, Office of Science, Office of Basic Energy Sciences, Division of Chemical Sciences, Geosciences, and Biosciences funded the work performed by LXD. The authors wish to thank the U.S. Department of Energy for funding the work through the Nuclear Energy University Program, NEUP Contract No. 120569. QNV acknowledges support from a Graduate Research Fellowship from the National Science Foundation (DGE1321846). The calculations were carried out using computer resources provided by the Office of Basic Energy Sciences. 


\section{References:}

1. Report of the American Nuclear Society President's Special Committee on Used Nuclear Fuel Management Options; 2011.

2. Blue Ribbon Commission on America's Nuclear Future. Report to the Secretary of Energy. U.S. Department of Energy: Washington DC; 2012.

3. H. Bay, Problems Concerning the Accumulation of Isolated Plutonium - the Swiss Situation; IAEA-TECDOC-765; 1994.

4. D. D, Sood and S. K. Patil. "Chemistry of Nuclear Fuel Reprocessing: Current Status." Journal of Radioanalytical and Nuclear Chemistry Articles 1996 203(2):547-573.

5. W. B. Lanham, T. C. Runion, "Purex Process for Plutonium and Uranium Recovery." 1949:1-13.

6. P. Guilbaud, G. Wipff. "Hydration of $\mathrm{UO}_{2}{ }^{2+}$ Cation and Its $\mathrm{NO}_{3}{ }^{-}$and 18-Crown-6 Adducts Studied by Molecular-Dynamics Simulations." Journal of Physical Chemistry 96 (1993) 5685.

7. S. Kerisit, C. Liu. "Structure, Kinetics, and Thermodynamics of the Aqueous Uranyl(VI) Cation." Journal of Physical Chemistry A 117 (2013) 6421.

8. S. P. Tiwari, N. E. Rai, E. J. Maginn. "Force Field Development for Actinyl Ions via Quantum Mechanical Calculations: An Approach to Account for Many Body Solvation Effects." Physical Chemistry Chemical Physics 16 (2014) 8060.

9. L. X. Dang, T. M. Chang. "Many-Body Interactions in Liquid Methanol and Its Liquid/Vapor Interface: A Molecular Dynamics Study.” Journal of Chemical Physics 119 (2003) 9851.

10. E. Guardia, R. Rey, J. A. Padro. Potential of Mean Force by Constrained MolecularDynamics - A Sodium-Chloride Ion-Pair in Water. Chemical Physics 155 (1991) 187.

11. Case, D. A., T. A. Darden, T. E. Cheatham III, C. L. Simmerling, J. Wang, R. E. Duke, R. Luo, K. M. Merz, D. A. Pearlman, M. Crowley, R. C. Walker, W. Zhang, B. Wang, S. Hayik, A. Roitberg, G. Seabra, K. F. Wong, F. Paesani, X. Wu, S. Brozell, V. Tsui, H. Gohlke, L. Yang, C. Tan, J. Mongan, V. Hornak, G. Cui, P. Beroza, D. H. Mathews, C. Schafmeister, W. S. Ross, P. A. Kollman. Amber 9. University of California, San Francisco: 2006.

12. U. Essmann, L. Perera, M. L. Berkowitz, T. Darden, H. Lee, L. G. Pedersen, A Smooth Particle Mesh Ewald Method. J. Chem. Phys. 103 (1995) 8577.

13. J. P. Ryckaert, G. Ciccotti, H. J. C. Berendsen, Numerical-Integration of Cartesian Equations of Motion of a System with Constraints Molecular Dynamics of N-Alkanes. J. Comp. Phys. 23 (1977) 327. 
14. D. G. Truhlar, W. L. Hase, J. T. Hynes, Current Status of Transition-State Theory. J. Phys. Chem. 87 (1983) 2664.

15. D. Chandler, Statistical-Mechanics of Isomerization Dynamics in Liquids and TransitionState Approximation. J. Chem. Phys. 68 (1978) 2959.

16. R. F. Grote and J.T. Hynes. "The Stable States Picture of Chemical-Reactions 2. Rate Constants for Condensed and Gas-Phase Reaction Models." Journal of Chemical Physics 73 (1980) 2715.

17. R.W. Impey, P.A. Madden, I.R. McDonald, Hydration and Mobility of Ions in Solution. J. Phys. Chem. 87, (1983) 5071.

18. L. Helm, A. E. Merbach, Inorganic and Bioinorganic Solvent Exchange Mechanisms. Chem. Rev. 105 (2005) 1923 and references cited therein.

19. I. Farkas, I. Bányai, Z. Szabó, I. Grenthe. "Rates and Mechanisms of Water Exchange of $\mathrm{UO}_{2}{ }^{2+}$ aq) and $\mathrm{UO}_{2}$ (oxalate)F- $\left(\mathrm{H}_{2} \mathrm{O}\right)^{2-}$ : A Variable-Temperature ${ }^{17} \mathrm{O}$ and ${ }^{19} \mathrm{~F}$ NMR Study." Inorganic Chemistry 39 (2000) 799.

20. M. Sprik. "Coordination numbers as reaction coordinates in constrained molecular dynamics” Faraday Discussions 110 (1998) 437. 
Table 1. Optimized potential parameters for water-water [9] and uranyl-water interactions used in the MD simulation. The terms $\sigma$ and $\varepsilon$ are the Lennard-Jones parameters, $q$ is atomic charge, and $\alpha$ is the molecular polarizability.

\begin{tabular}{ccccc}
\hline Atom Type & $\sigma(\AA)$ & $\varepsilon(\mathrm{kcal} / \mathrm{mol})$ & $\mathrm{q}(\mathrm{e})$ & $\alpha\left(\AA^{3}\right)$ \\
\hline $\mathrm{O}$ & 3.2215 & 0.1825 & 0.0000 & 0.0000 \\
$\mathrm{H}$ & 0.0000 & 0.0000 & 0.5190 & 0.0000 \\
$\mathrm{M}$ & 0.0000 & 0.0000 & -1.0380 & 1.4440 \\
$\mathrm{U}$ & 3.3498 & 0.0270 & 3.1250 & 1.0000 \\
$\mathrm{O}(\mathrm{U})$ & 3.2963 & 0.4380 & -0.5625 & 0.0000 \\
\hline
\end{tabular}

Table 2. Hydration properties of the $\mathrm{UO}_{2}{ }^{2+}-\mathrm{H}_{2} \mathrm{O}$.

\begin{tabular}{rccc}
\hline & Coordination Number & Hydration Enthalpy & First Maximum \\
\hline MD & $5.0 \pm 0.1$ & $-406 \pm 3 \mathrm{kcal} / \mathrm{mol}$ & $2.40 \pm 0.05 \AA$ \\
Expt. & $4.9 \pm 0.4$ & -397 to $418 \mathrm{kcal} / \mathrm{mol}$ & 2.40 to $2.42 \AA$ \\
\hline
\end{tabular}

Table 3: Rate theory results

\begin{tabular}{cccccc}
\hline $\mathrm{UO}_{2}{ }^{2+}$ & Pressure $/$ bar & $k^{\mathrm{TST}} / \mathrm{ps}$ & $k^{\mathrm{TST}} \square_{\mathrm{RF}} / \mathrm{ps}$ & $k^{\mathrm{TST}} \square_{\mathrm{GH}} / \mathrm{ps}$ & $k_{\mathrm{IMM}} / \mathrm{ps}$ \\
\hline 0 & $1.48 \times 10^{-4}$ & $3.1 \times 10^{-5}$ & $3.6 \times 10^{-5}$ & $6.8 \times 10^{-5}$ \\
2000 & $1.09 \times 10^{-4}$ & $2.4 \times 10^{-5}$ & $1.6 \times 10^{-5}$ & $3.7 \times 10^{-5}$ \\
4000 & $0.85 \times 10^{-4}$ & $2.0 \times 10^{-5}$ & $1.1 \times 10^{-5}$ & $3.4 \times 10^{-5}$
\end{tabular}

Note: The estimated error in transmission coefficients is \pm 0.01 . 


\section{Figure Captions:}

Figure 1. Computed rate density function and corresponding running coordination number for U$\mathrm{O}$ at ambient conditions.

Figure 2. Computed PMFs for $\mathrm{UO}_{2}{ }^{2+}-\mathrm{H}_{2} \mathrm{O}$ pair at three different pressures.

Figure 3. Computed time-dependent transmission coefficients, $\quad(t)$, of $\mathrm{UO}_{2}{ }^{2+}-\mathrm{H}_{2} \mathrm{O}$ pair in water from the RF method at three different pressures.

Figure 4. Computed time-dependent friction kernels, $(t)$, of $\mathrm{UO}_{2}{ }^{2+}-\mathrm{H}_{2} \mathrm{O}$ pair in water from the $\mathrm{GH}$ theory at three different pressures.

Figure 5. Computed time-correlation function, $P(t)$, as a function of pressure used to determine the residence time of an $\mathrm{H}_{2} \mathrm{O}$ molecule is in the first solvation shell of the $\mathrm{UO}_{2}{ }^{2+}$ at three different pressures. 
Figure 1.

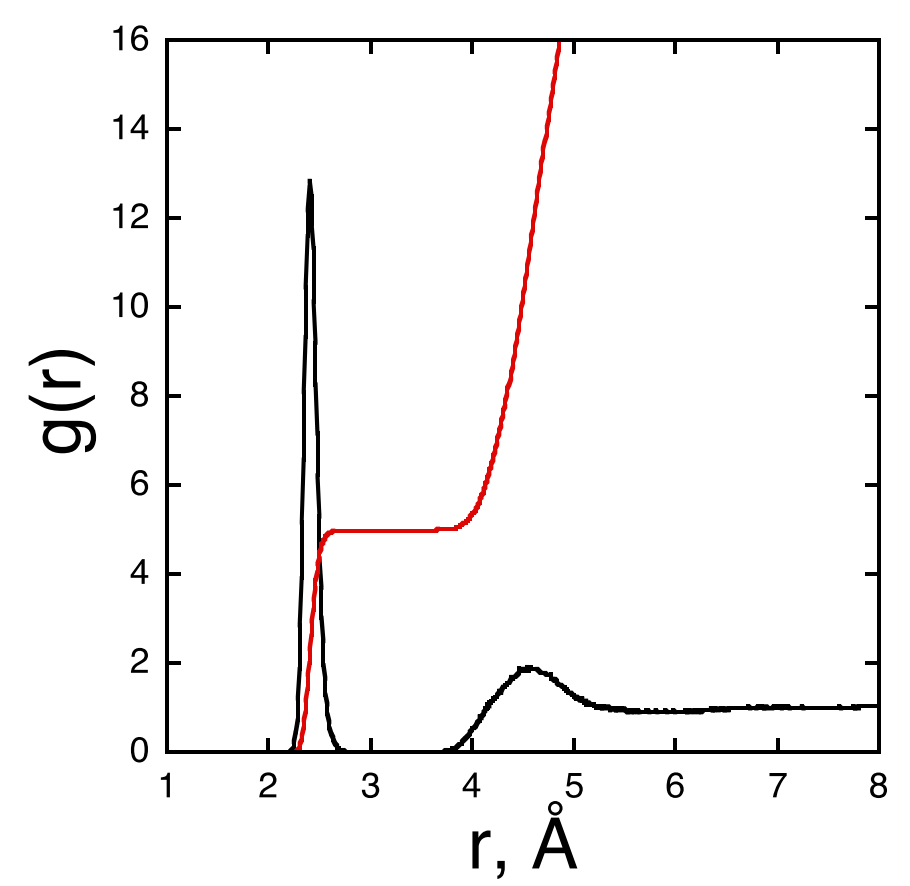

Figure 2.

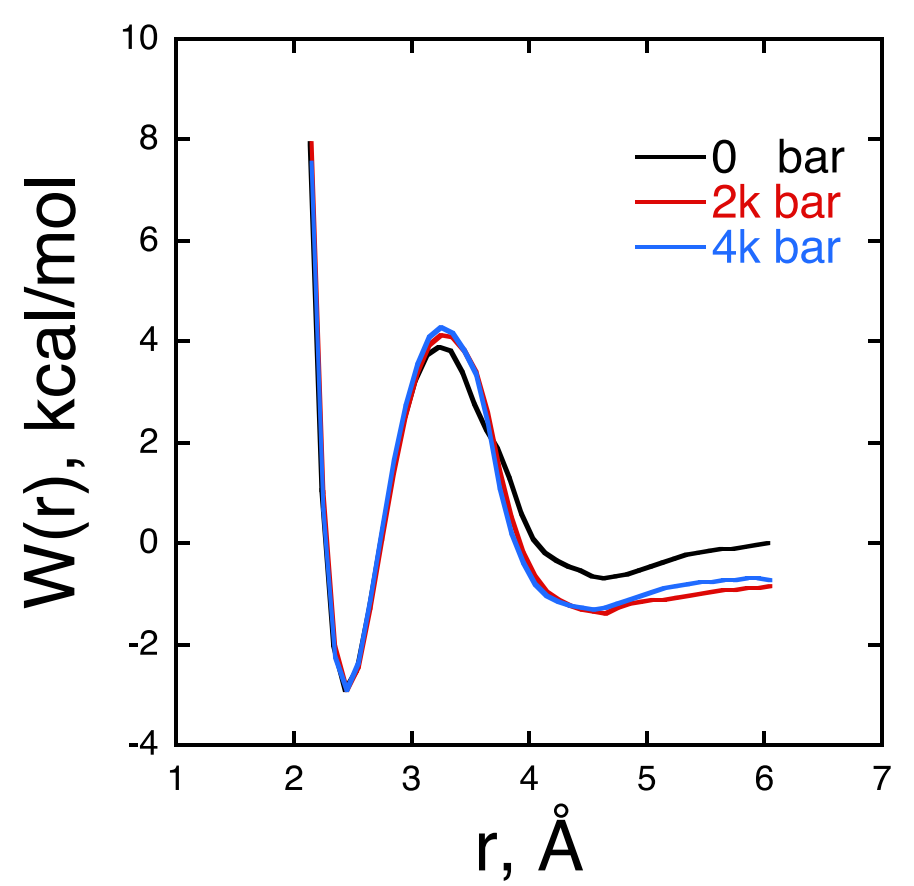


Figure 3.

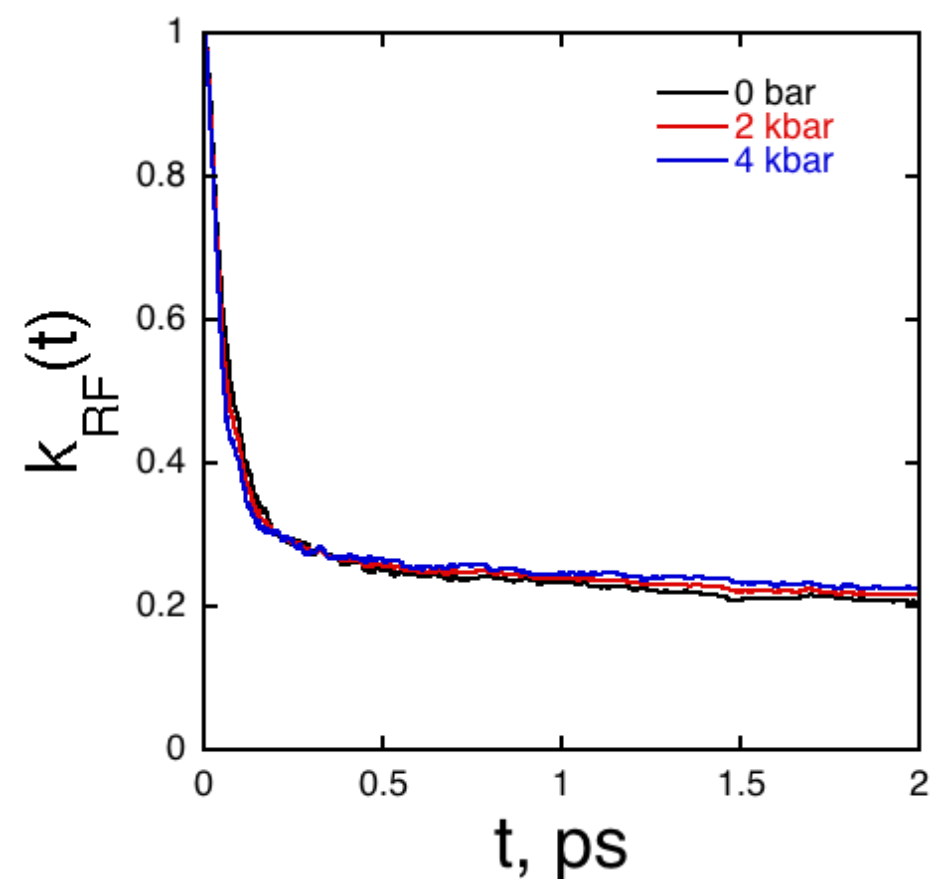

Figure 4.

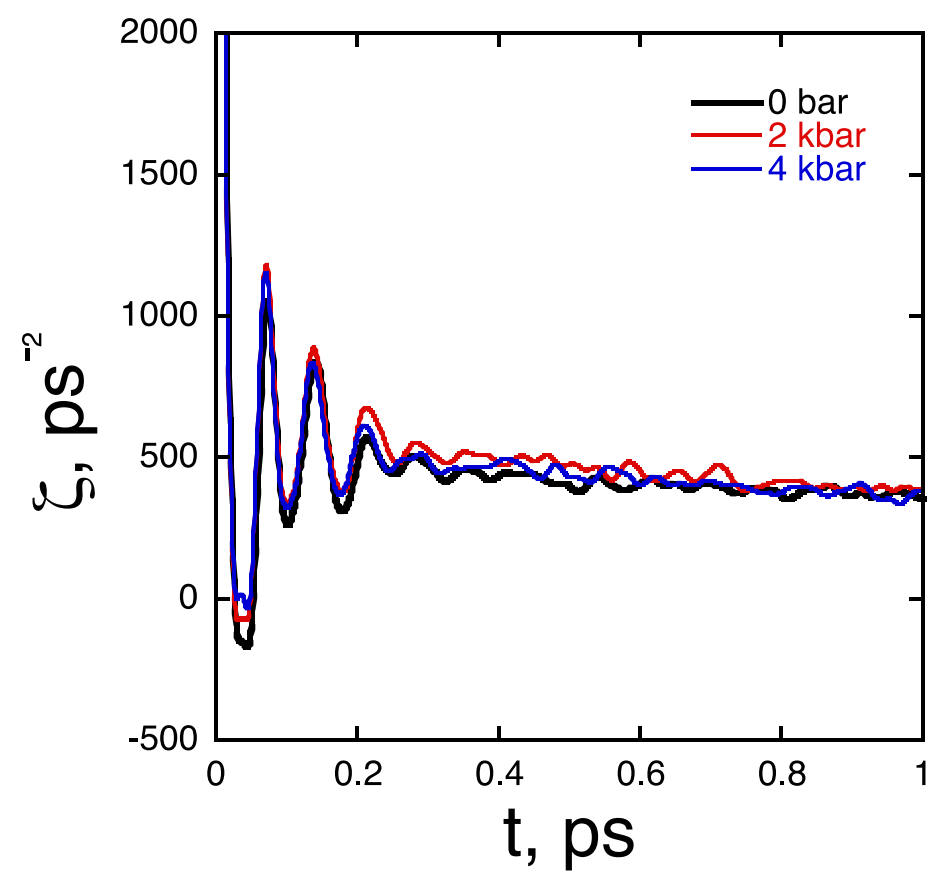


Figure 5.

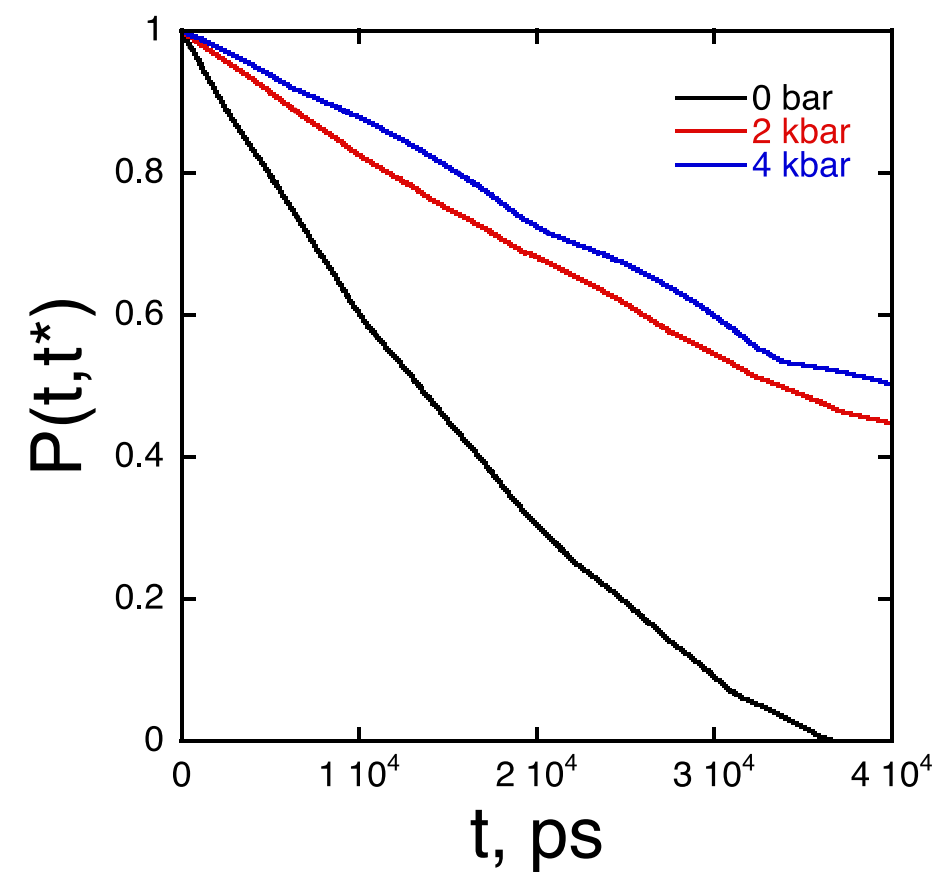

